\title{
The LMC Centre Unfolding
}

\author{
A. Ardeberg, P. Linde \\ Lund Observatory, Box 43, SE-221 00 Lund, Sweden
}

\section{B. Gustafsson}

Uppsala Astronomical Observatory, Box 515, S-751 20 Uppsala, Sweden

\begin{abstract}
We study star formation history and chemical evolution in the LMC Bar centre. With the HST PC and WFC plus uvby photometry, we find $30 \%$ of the stars younger than $200 \mathrm{My}$ and evidence for reduced star formation 3 to $0.2 \mathrm{~Gy}$ ago. The overall $[\mathrm{Fe} / \mathrm{H}]$ is -0.5 . Many stars older than $4 \mathrm{~Gy}$ contradict a very young Bar.
\end{abstract}

\section{Introduction}

In the Bar of the Large Magellanic Cloud (LMC), stellar density and distance have prevented safe studies of star formation, stellar abundance and evolution. Image crowding prohibited precision turn-off photometry in colour magnitude diagrams (CMD), with spectroscopic abundance studies feasible for the brightest stars only. These data shed little light on star formation and chemical evolution.

\section{Star formation and metallicity}

The Hubble Space Telescope (HST) and its planetary and wide field camera (WFPC2) dramatically improve our studies of individual faint LMC Bar stars. This is due to the high image quality and combined PC and WFC image scales and fields of view. With the HST PC and uvby photometry, we derived a detailed CMD and metal abundances for the centre of the LMC Bar. We studied individual stars with $\mathrm{V}(\mathrm{y})<24$ (Ardeberg et al. 1997, 1999).

The PC study showed star formation over up to 9 Gy. It gave proof that close to $30 \%$ of the stars in the Bar centre are less than $500 \mathrm{My}$. There was evidence of a halt in star formation starting at least 2 Gy ago and ending with the recent burst of star formation. More exact determinations were impossible due to weak statistics of brighter stars, a consequence of the small PC field.

Since our first studies, based on PC data only, we added data from one WFC field. It covers $6400 \operatorname{arcsec}^{2}$, as compared to the PC field of $1200 \operatorname{arcsec}^{2}$. By combining PC and WFC data, we can profit from best spatial resolution, highest photometric accuracy and favourable number statistics.

For the luminosity function, we developed a new method, including stars individually unidentified but statistically measurable. The LF agrees well with the solar vicinity one, even in details. See Fig. 1, left. It is still rising at V=26. In both respects, it resembles the LF of LMC structures outside the Bar. 

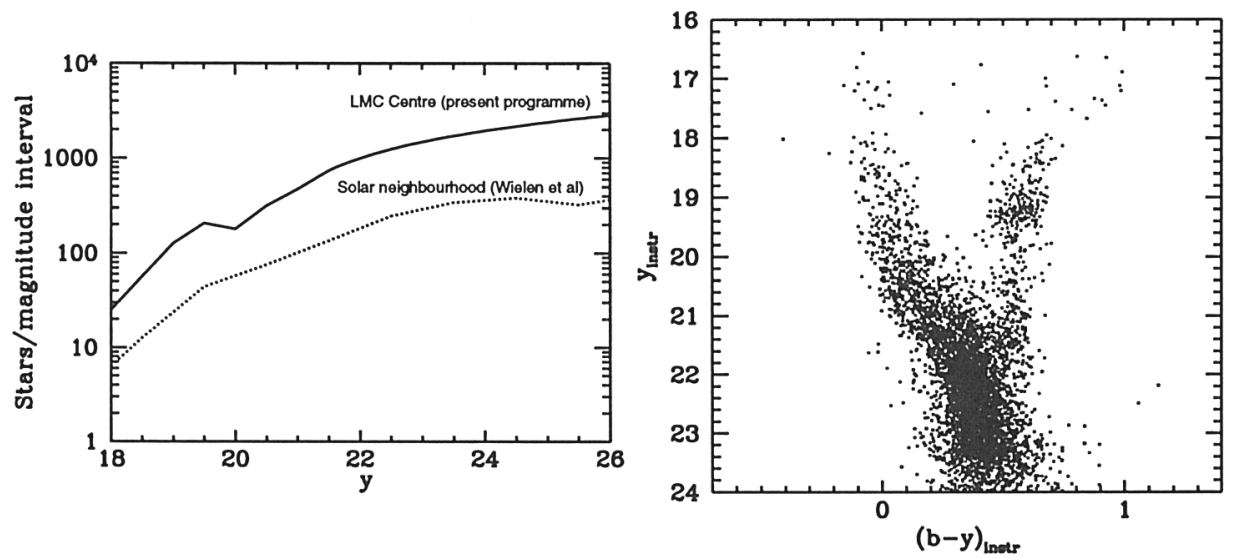

Figure 1. Left: Luminosity function compared to solar neighbourhood. Right: CMD from WF3 field. 6000 stars are included.

Fig. 1 (right) shows a tentative CMD. Of somewhat lower quality compared to PC data, for the brighter stars the WFC has important advantages. Comprising 6000 stars brighter than $\mathrm{V}(\mathrm{y})=24$, the WFC CMD presents far better statistics than the PC CMD for $\mathrm{V}(\mathrm{y})<22$. The improvement is even larger for stars around and above the upper turn-off region. Noteworthy is the tip of the main sequence as is the clump and the CMD morphology. The combined PC and WFC data improve population definition and star formation history, especially for stars younger than 3-4 Gy. With our new data, using the techniques described in Ardeberg et al. (1997), we improve our estimate of the upper age limit for the youngest population from 500 to $200 \mathrm{My}$. We confirm that $30 \%$ of the stars belong to this population. We improve the lower age limit of reduced star formation from 2 to $3 \mathrm{~Gy}$. The improved CMD makes our morphology based estimate of metallicity safer while remaining at $[\mathrm{Fe} / \mathrm{H}]=-0.5$, close to the average result from our $\mathrm{m}_{1}$ versus (b-y) data obtained from $\mathrm{PC}$ observations. The latter data point to an evolutionary trend in $[\mathrm{Fe} / \mathrm{H}]$ over the latest $6 \mathrm{~Gy}$, an evolution so far unknown to any detail.

\section{Concluding remarks}

Our results are highly relevant for the lively discussion of the evolution of the LMC Bar. We find little support for the current tendency (van den Bergh, in this volume) to classify the Bar as a young part of the LMC. This is contradicted by both our age determinations of stellar populations and by the trend in $[\mathrm{Fe} / \mathrm{H}]$ noted for the same stars. Further reduction and analysis will improve our CMD statistics and $[\mathrm{Fe} / \mathrm{H}]$ data, giving us a better handle on evolution.

\section{References}

Ardeberg, A., Gustafsson, B., Linde, P., \& Nissen, P.-E., 1997, A\&A, 322, L13 Ardeberg, A., et al. 1999, in IAU Symp. No. 184, in press 